\title{
A Multi-Band WCDMA SAW-Less Receivers with Frequency Selective Feedback Loop
}

\author{
Sungho Beck, Stephen T. Kim, Kyutae Lim, \\ and Manos M. Tentzeris \\ Dept. of Electrical and Computer Engineering \\ Georgia Institute of Technology \\ Atlanta, Georgia, USA
}

\begin{abstract}
For frequency division duplex (FDD) wide band code division multiple access (WCDMA) system, transmit (TX) signal leakage at the receiver input degrades the receiver performance. Previously, to cancel the TX leakage, a frequency selective feedback was proposed, but it had the unpredictable feedback loop characteristic due to the uncertainty of the duplexer impedance. This paper proposes a new method to achieve a predictable feedback loop characteristic. In addition, to achieve the TX leakage cancellation for multi-band, a local oscillator (LO) phase shift technique is also presented. The proposed receiver which was composed of high- and low-band LNAs and mixers, the TX canceller, and the LO shifter was implemented using $0.18-\mu \mathrm{m}$ CMOS process and is under fabrication. Simulation results met the required specifications which were delivered from the WCDMA specifications.
\end{abstract}

\section{INTRODUCTION}

Because of the high data rate possibility of the wireless code division multiple access (WCDMA) terminals, multiple bands have been adopted for the WCDMA standard, e.g., ten bands are allocated for the WCDMA standard [1]. To provide cheap and small multi-band mobile terminals, a low cost multi-band receiver with a small PCB form factor has been demanded. Due to improvements of the CMOS process, active blocks of the receiver such as low-noise amplifiers (LNAs) and mixers are ready to support multi-band without introducing significant penalty of cost and PCB area. However, external bulky and expensive standing acoustic wave (SAW) filters have been used to attenuate transmit (TX) signals. A strong TX signal co-exists with the weak receive (RX) signal in the frequency division duplex (FDD) WCDMA system, and it distorts the RX signal [2]. Since an SAW filter is required for each additional RF band, SAW filters become the obstacle of the realization of cheap and small multi-band receivers.

To solve this problem, researches have been actively performed as [2]-[4]. In [3], the author utilized a feed-forward cancellation technique to realize band-pass characteristic. However, it is sensitive to the mismatch between the main path and the feed-forward path. In [2], the TX leakage was attenuated by a sinker after a down-conversion mixer. In contrast to [3], this technique does not suffer from the path mismatch, but it requires addition local oscillator (LO) signal

\author{
Joy Laskar
}

and a highly linear down-conversion mixer. In [4], feedback was adopted to achieve TX leakage cancellation, but it has a problem of the uncertainty in the feedback loop. The reason of the feedback uncertainty will be presented in the following chapter. In addition, all of the above researches focused only one frequency band.

A new feedback method with predictable feedback loop characteristic for the TX leakage cancellation will be presented in this paper, and a method for the multi-band application will be also presented.

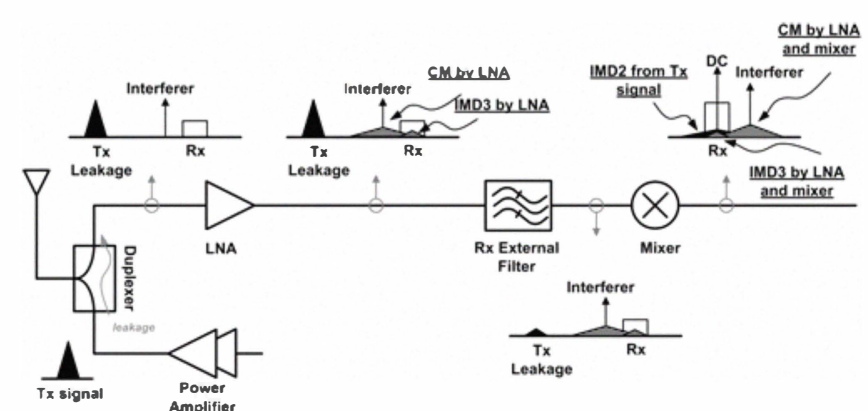

(a)

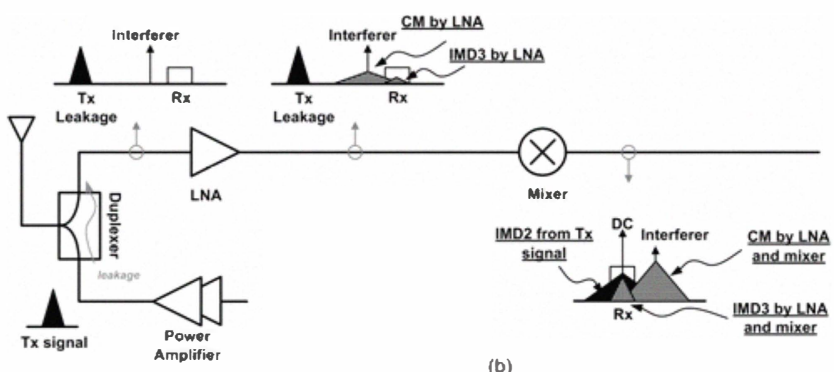

(b)

Figure 1. Influence of the TX leakage to the receiver performance, (a) with a SAW filter, (b) without a SAW filter 


\section{LINK BUDGET FOR WCDMA SAW-LESS RECEIVERS}

A. Influence of the TX leakage to the receiver performance

A duplexer has been used to isolate a weak RX signal from the strong TX signal as Fig. 1. However, because of limited isolation between the TX port and the RX port of the duplexer, typically $50 \mathrm{~dB}$, TX leakage exists at the LNA input [5]. In addition, other interferers may arrive at the antenna. The major distortion mechanisms are explained as follows:

1) Second-order inter-modulation (IMD2): The secondorder distortion of the modulated TX leakage in the mixer generates disturbance around $D C$, where the $R X$ signal is down-converted. The quantitive expression is Input referred second-order intercept point (IIP).

2) Third-order inter-modulation (IMD3) or crossmodulation (CM): The TX leakage and another jammer may generate distortions by third-order nonlinearity. The quantitive expression is input referred thord-order intercept point (IIP 3 ).

Because the jammers and the TX leakage are amplified by the LNA, the mixer must have exceptionally high $\mathrm{IIP}_{2}$ and $\mathrm{IIP}_{3}$ if they are not attenuate before the mixer. The attenuation of the out-of-the band jammers and the TX leakage is the role the SAW filter in the WCDMA receiver.

\section{B. Required receiver performance}

Based on the WCDMA standard of [1], the receiver specifications are derived as Table I using similar method of [2].

\section{Required TX attenuation for the SAW-less receiver}

To meet the specifications of Table I, required out-of-the band $\mathrm{IIP}_{2}\left(\mathrm{IIP}_{2, \mathrm{OB}}\right)$ and $\mathrm{IIP}_{3}\left(\mathrm{IIP}_{3, \mathrm{OB}}\right)$ of the mixer are calculated using Equation (1) and (2) when a SAW filter is used.

$$
\begin{aligned}
& I I P_{2, O B}=I I P_{2, \text { mixer }}+I L-G_{L N A}+2 A_{O B}, \\
& \frac{1}{I I P_{3, O B, \text { linear }}}=\frac{1}{I I P_{3, L N A, \text { linear }}}+\frac{G_{L N A, \text { linear }} / I L_{\text {linear }}}{I I P_{3, \text { mixer,linear }}} \frac{1}{10^{3 A_{O B} / 40}},
\end{aligned}
$$

in which, $I L, G_{L N A}$, and $A_{O B}$ stand for the insertion loss of the SAW filter, the gain of LNA, and the out-of-the band attenuation of the SAW filter, respectively. On the other hand, if only the TX leakage is attenuated, the $\mathrm{IIP}_{3, \mathrm{OB}}$ requirement is changed as Equation (3) in which $\mathrm{A}_{\mathrm{TX}}$ means the amount of the attenuation of the TX leakage

$$
\frac{1}{I I P_{3, \text { OB,linear }}}=\frac{1}{I I P_{3, L N A, \text { linear }}}+\frac{G_{L N A, \text { linear }} / I L_{\text {linear }}}{I I P_{3, \text { mixer,linear }}} \frac{1}{10^{2 A_{T X} / 40}} \text {. }
$$

Note that the exponent is 2 in Equation (3) instead of 3 in Equation (2). This comes from the fact that only the TX leakage is attenuated, while the TX leakage and another jammer are attenuated by the SAW filter in Equation (2). That means more TX leakage attenuation that the out-of-the band attenuation of the SAW filter to achieve the same LNA input referred $\mathrm{IIP}_{3, \mathrm{OB}}$. The required mixer $\mathrm{IIP}_{2, \mathrm{OB}}$ and $\mathrm{IIP}_{3, \mathrm{OB}}$ to satisfy Table I are plot versus the TX attenuation in Fig. 2.
With more than 20-dB TX cancellation, the mixer performances are relaxed to the feasible range. The required LNA and mixer performances with the 20-dB TX attenuation are summarized in Table II comparing with the case of adopting a conventional SAW filter [5]

TABLE I. WCDMA RECEIVER SPECIFICATIONS

\begin{tabular}{c|c|c|c}
\hline \hline & Unit & Spec. & Main source \\
\hline $\mathrm{NF}$ & $\mathrm{dB}$ & 3 & Thermal noise \\
$\mathrm{IIP}_{2, \mathrm{IB}}$ & $\mathrm{dBm}$ & 3 & In-band blocker \\
$\mathrm{IIP}_{2, \mathrm{OB}}$ & $\mathrm{dBm}$ & 50 & TX leakage \\
$\mathrm{IIP}_{3, \mathrm{IB}}$ & $\mathrm{dBm}$ & -21 & Inter-modulation \\
$\mathrm{IIP}_{3, \mathrm{OB}}$ & $\mathrm{dBm}$ & -5 & TX and double duplex \\
\hline \hline
\end{tabular}

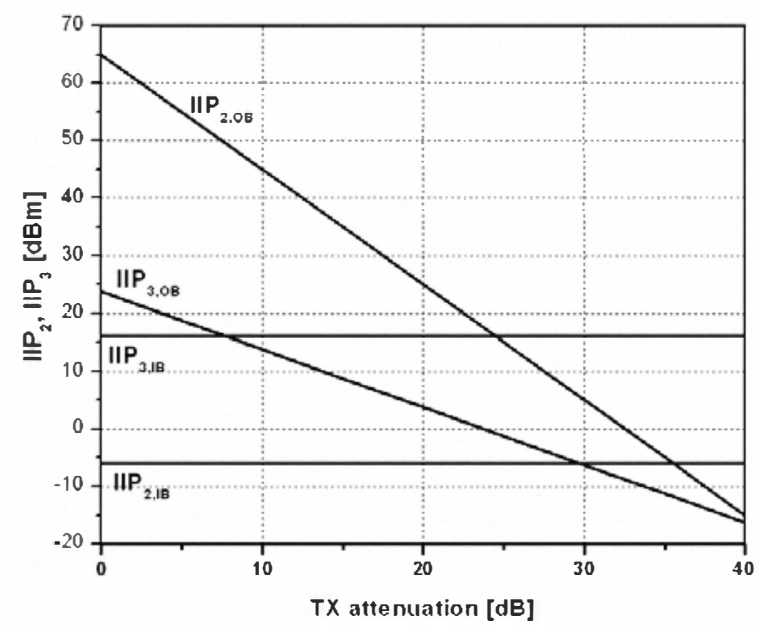

\begin{tabular}{|c|c|c|c|c|}
\hline \multicolumn{5}{|c|}{ (a) With commercial SAW filter [5] } \\
\hline & Unit & LNA & Mixer & Total \\
\hline Gain & $\mathrm{dB}$ & 15 & 20 & 33 \\
\hline NF & $\mathrm{dB}$ & 1.5 & 10 & 2.75 \\
\hline $\mathrm{IIP}_{2, \mathrm{IB}}$ & $\mathrm{dBm}$ & - & 16 & 3 \\
\hline IIP $_{2, \mathrm{OB}}$ & $\mathrm{dBm}$ & - & -1 & 50 \\
\hline $\mathrm{IIP}_{3, \mathrm{IB}}$ & $\mathrm{dBm}$ & -3 & -8 & -3 \\
\hline $\mathrm{IIP}_{3, \mathrm{OB}}$ & $\mathrm{dBm}$ & -3 & -26 & -5 \\
\hline \multicolumn{5}{|c|}{ (b) With 20-dB TX attenuation $(\mathrm{NF}=0.5 \mathrm{~dB})$} \\
\hline & Unit & LNA & Mixer & Total(IB/OB) \\
\hline Gain & $\mathrm{dB}$ & 15 & 20 & 35 \\
\hline NF & $\mathrm{dB}$ & 1.5 & 10 & 2.72 \\
\hline $\mathrm{IIP}_{2}$ & $\mathrm{dBm}$ & - & 25 & $10 / 50$ \\
\hline $\mathrm{IIP}_{3}$ & $\mathrm{dBm}$ & -3 & 4 & $-12 /-5$ \\
\hline
\end{tabular}

Figure 2. Required mixer linearity performances vs. the TX attenuation

TABLE II. LNA AND MIXER SPECIFICATIONS 


\section{PROPOSED TX CANCEllation Method}

\section{A. Uncertain out-of-the band impedance of the duplexer}

Reference [4] proposed to close the feedback loop by supply the TX cancellation signal to the joint of the LNA input and the duplexer RX port. The feedback loop characteristics such as the loop gain and the phase margin depend on the impedance of the joint. However, the RX port impedance of the duplexer is unknown as Fig. 3 [6]. Although the RX port impedance is well-defined in the RX frequency to $50 \Omega$, the reflection coefficient is placed at the edge of the Smith chart in the TX frequency in which the feedback loop is closed. Therefore, the RX port impedance in the TX frequency varies from short to open along with the distance between the duplexer and the receiver. Therefore, the characteristics of the feedback become uncertain, and it has potential problems.

\section{B. Proposed frequency selective feedback method}

To overcome this problem, a new technique is proposed to connect the feedback loop to the LNA output load as Fig. 4. By isolating the feedback loop from the duplexer, the characteristics of the feedback becomes predictable. Indeed, as the feedback is formed only inside the chip, it is insensitive to the variations of external components.

First, the RX signal part is designed as follows. To reduce pin counts and external components comparing to the previous approach [4], the LNA has a single-ended input. The LNA is consists of a common-source trans-conductance stage followed by a common-gate cascode stage that improves the RX LO leakage at the LNA input and a tuned load with an inductor and capacitors. Then, following RX passive mixers convert the RX signal to DC. Passive mixers are used to improve the linearity of the mixer [7]. Low band around 1$\mathrm{GHz}$ and high band around $2-\mathrm{GHz}$ are integrated in a single chip as the WCDMA bands are allocated from $700 \mathrm{MHz}$ to $2.6 \mathrm{GHz}$.

Second, the TX leakage cancellation part is designed as follows. By adopting TX LO signals which already exist in TX blocks, the TX leakage at the LNA output is downconverted to DC by first passive mixers. The down-converted $\mathrm{RX}$ signal which lies at the duplexer frequency, $190-\mathrm{MHz}$ for the band-I [1], is attenuated by simple one-pole low-pass filters (LPFs), but the TX leakage around DC is not attenuated by the LPFs. Finally, the TX signal at DC is restored to the original TX frequency by up-conversion mixers. Then, the TX signal is fed to the LNA load and cancels the TX signal from the LNA. By attenuating the RX signal in the feedback loop, the loop gain of the RX signal becomes negligible not to affect the RX signal. Since the TX leakage cancellation occurs at the output of the LNA, the LNA still needs high linearity. However, LNA linearization methods have been used as [8].

To achieve largest phase margin over multi-band, the loop phase at the TX center frequency should be $180^{\circ}$ for all the bands. However, the phase around the feedback loop depends on the RF frequency as the time delay in the RF block shifts phase of the loop [4]. Therefore, to use the single TX canceller for multi-band, a method to change the feedback loop phase according to each band is required.

\section{LO phase shifter for mult-band application}

If the inputs of the TX canceller, TX_LO1, and TX_LO2 are presented as $\cos \left(\omega_{i n} t\right), \cos \left(\omega_{L O} t+\phi\right)$, and $\cos t\left(\omega_{L O} t+\theta\right)$, respectively, the output of the TX canceller is $\cos \left(\omega_{i n} t+\phi-\theta\right)$ with normalization in the amplitude. Therefore, to change the phase of the TX canceller output- $\phi-\theta$, hence the phase of the feedback loop, the phase of TX LO1 or TX_LO2 should be adjusted independently. To rotate the phase of TX_LO1 with respect to TX LO2, a phase shifter is designed as Fig. 5 [9]. By adding two quadrature signals with adjustable strength, $\alpha$, the LO phase can be changed. As thermal noise from the phase shifter can be filtered by the LPF in the TX canceller, the phase of LO1, not LO2, are shifted.

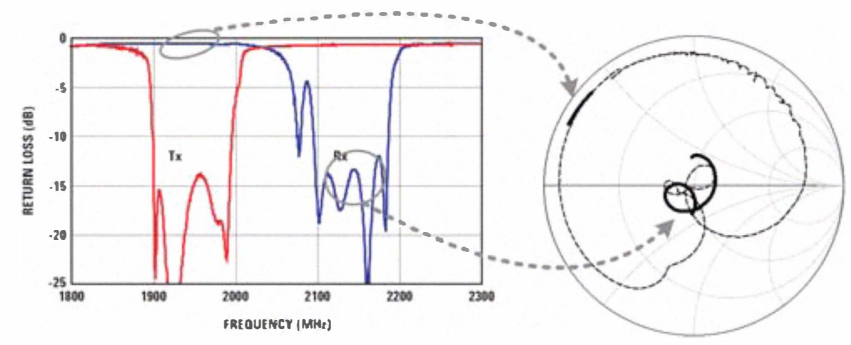

Figure 3. Duplexer RX port impedance

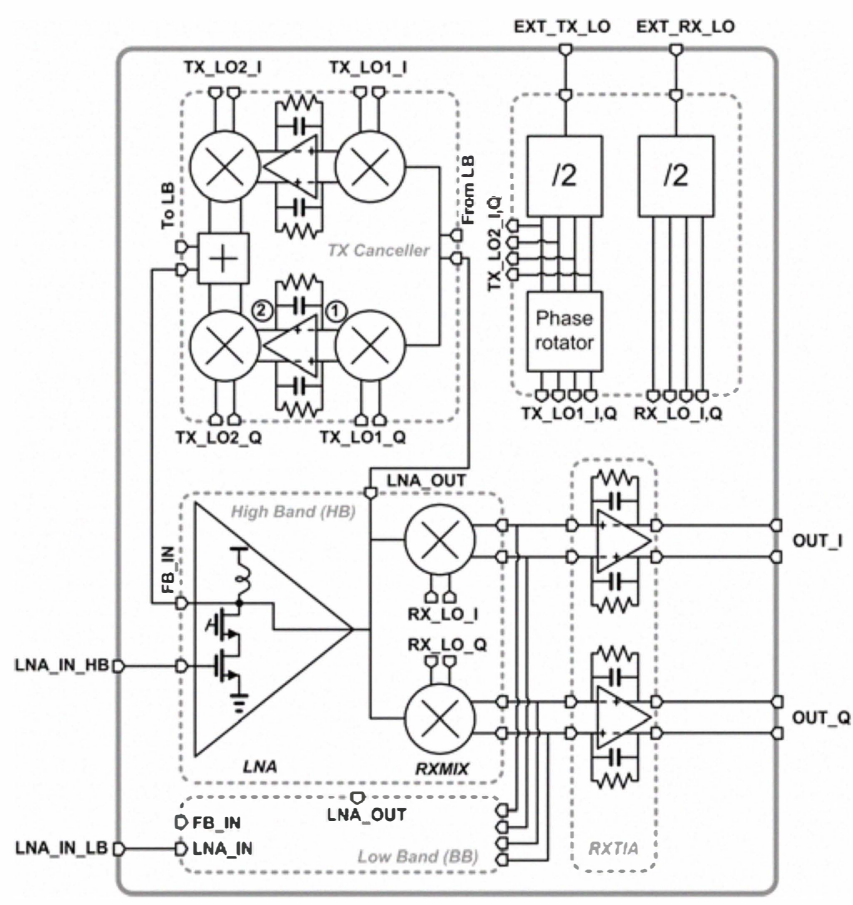

Figure 4. Proposed multi-band receiver with TX leakage cancellation.

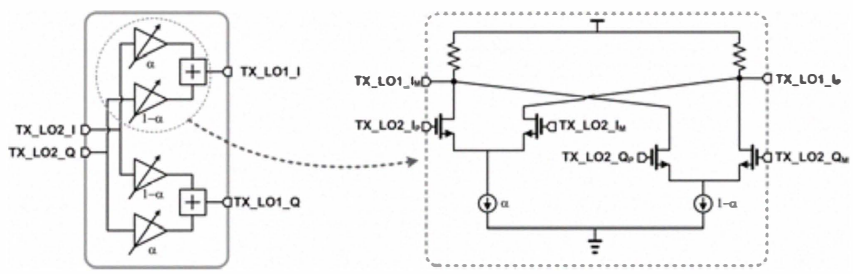

Figure 5. LO phase rotator 


\section{SIMULATION RESULTS AND IMPLEMENTATION}

The proposed SAW-less receiver is implemented using $0.18-\mu \mathrm{m}$ CMOS process as Fig. 6 . The area was $2.2 \mathrm{~mm}^{2}$ and it is under the fabrication. Fig. 7 shows the frequency responses of the receiver at the LNA outputs and the mixer outputs for both bands with change of LO phase. The TX leakage attenuation of $30 \mathrm{~dB}$ and $27 \mathrm{~dB}$ were achieved for the low band and high band, respectively. Fig. 8 shows the noise figure (NF) of the LNAs only and the total receivers. NF degradation for the low band and the high band were $0.7 \mathrm{~dB}$ and $0.5 \mathrm{~dB}$, respectively. The comparison of the performance to previous works is summarized in Table III. This work is superior to [2] with respect to smaller NF degradation and low power consumption, but inferior to [4]. However, as explained in the section III-A, [4] has a potential problem of the feedback loop uncertainty.

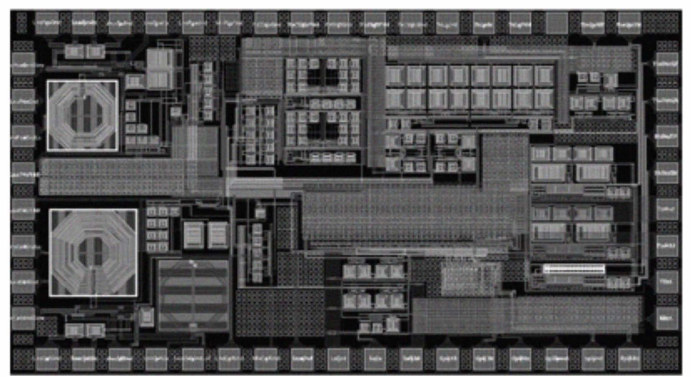

Figure 6. Capture of the layout.
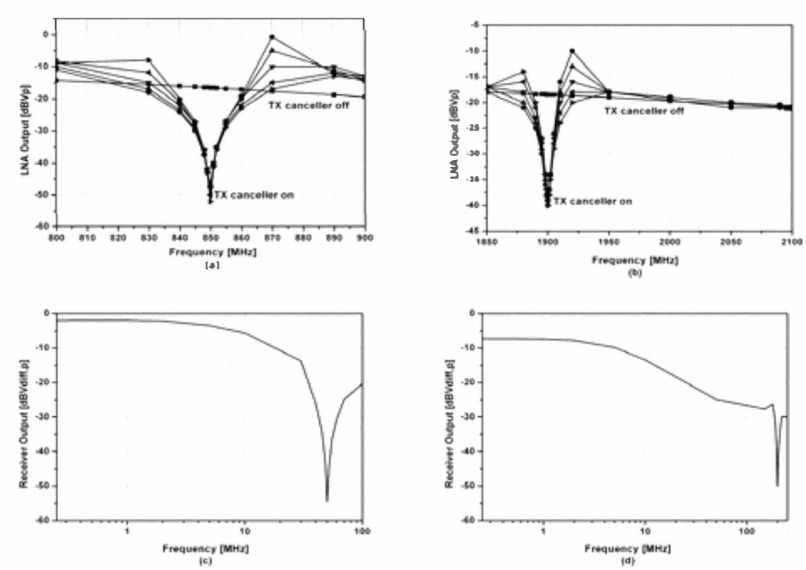

Figure 7. Frequency response of the receiver (a) LB LNA out, (b) HB LNA out, (c) LB RX total, (d) HB RX total.

TABLE III. PERFORMANCE COMPARISON TO PREVIOUS WORKS

\begin{tabular}{c|c|c|c|c|c}
\hline \hline & & \multirow{2}{*}[2]{} & \multirow{2}{*}[4]{$^{\mathrm{a}}$} & \multicolumn{2}{|c}{ This work } \\
\cline { 5 - 6 } & & & & LB & HB \\
\hline \multirow{2}{*}{ Block } & & LNA & \multirow{2}{*}{ LNA } & \multicolumn{2}{|c}{ LNA + Mixer } \\
& & Mixer & & & \multicolumn{2}{|c}{} \\
Gain & $\mathrm{dB}$ & 45 & 19.9 & 36 & 33.7 \\
NF $^{\mathrm{b}}$ & $\mathrm{dB}$ & $3.1 / 4.8$ & $1.5 / 1.7$ & $1.4 / 2.1$ & $1.4 / 1.9$ \\
Tx Att. & $\mathrm{dB}$ & 6 & 18 & 30 & 27 \\
Current & $\mathrm{mA}$ & $30 / 53$ & $11 / 15$ & $13 / 20$ & $16 / 23$ \\
\hline
\end{tabular}

a) Feedback loop uncertainty, b) TX canceller off/on
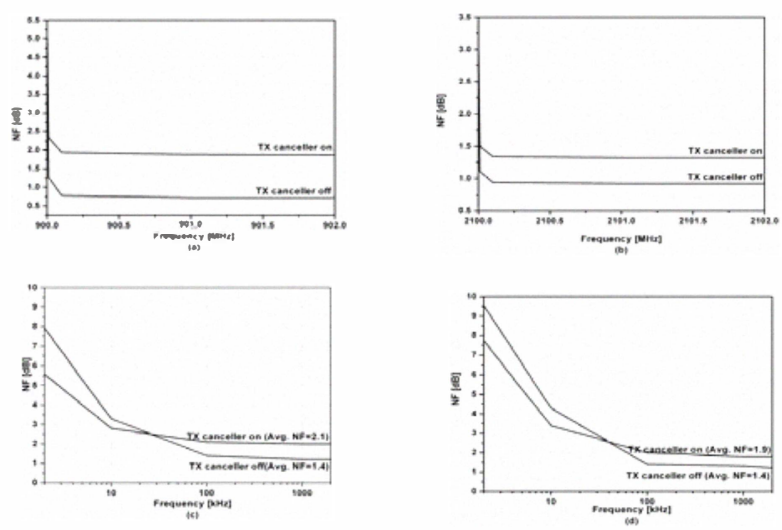

Figure 8. Noise figure of the receiver (a) LB LNA out, (b) HB LNA out, (c) LB RX total, (d) HB RX total.

\section{CONCLUSION}

This paper has proposed a new method for the TX leakage cancellation for a WCDMA receiver. Required TX leakage attenuation, more than $20 \mathrm{~dB}$, was delivered from the WCDMA standard, and frequency selective feedback was used to accomplish this TX leakage attenuation. By completing the feedback loop inside the chip, the feedback loop characteristic was predictable and insensitive to the duplexer impedance. To support multi-band, a LO phase shifter was also integrated to achieve maximum phase margin for each band. The proposed receiver was implemented using $0.18-\mu \mathrm{m}$ CMOS process. The simulation results met the standard specifications and showed the effectiveness of the proposed technique.

\section{REFERENCES}

[1] Universal Mobile Telecommunications System (UMTS); User Equipment (UE) conformance specification; Radio transmission and reception (FDD); Part 1: Conformance specification (3GPP TS 34.1211 version 8.8.0 Release 8).

[2] H. Khatri, P. S. Gudem, L. E. Larson, "An Active Transmitter Leakage Suppression Technique for CMOS SAW-Less CDMA Receivers," Solid-State Circuits, IEEE Journal of, vol. 45, no. 8, pp. 1590-1601, Aug. 2010.

[3] H. Darabi, "A Blocker Filtering Technique for SAW-Less Wireless Receivers," Solid-State Circuits, IEEE Journal of, vol. 42, no. 12, pp. 2766-2773, Dec. 2007.

[4] V. Aparin, "A New Method of TX Leakage Cancellation in W/CDMA and GPS Receivers," in IEEE Radio Frequency Integrated Circuits Symposium, 2008, pp. 87-90.

[5] Epcos SAW filter: B9411. Datasheet online available at http://www.epcos.com/inf/40/ds/mc/B9411.pdf.

[6] Avago Technologies FBAR duplexer: ACMD 7612. Datasheet online available at http://www.avagotech.com/docs/AV02-0473EN.

[7] J. Crols, M. S. J. Steyaert, "A $1.5 \mathrm{GHz}$ Highly Linear CMOS Downcoversion Mixer," Solid-State Circuits, IEEE Journal of, vol. 30, no. 7, pp. 736-742, F 1995.

[8] V. Aparin and L. E. Larson, "Modified Derivative Superposition Method for Linearizing FET Low-Noise Amplifier," in Microwave Theory and Techniques, IEEE Transactions on, vol. 53, no. 2, pp. 571581, Feb. 2005.

[9] L. Der, B. Razavi, "A 2-GHz CMOS Image-Reject Receiver With LMS Calibration," Solid-State Circuits, IEEE Journal of, vol. 38, no. 2, pp. 167-175, Feb. 2003. 